\title{
Fish Intake and Risk of Incident Atrial Fibrillation
}

\section{Citation}

Mozaffarian, D.. 2004. 'Fish Intake and Risk of Incident Atrial Fibrillation.' Circulation 110, 4:

368-373. https://doi.org/10.1161/01.CIR.0000138154.00779.A5

\section{Permanent link}

http://nrs.harvard.edu/urn-3:HUL.InstRepos:41249122

\section{Terms of Use}

This article was downloaded from Harvard University's DASH repository, and is made available under the terms and conditions applicable to Other Posted Material, as set forth at http:// nrs.harvard.edu/urn-3:HUL.InstRepos:dash.current.terms-of-use\#LAA

\section{Share Your Story}

The Harvard community has made this article openly available.

Please share how this access benefits you. Submit a story.

Accessibility 


\title{
Fish Intake and Risk of Incident Atrial Fibrillation
}

\author{
Dariush Mozaffarian, MD, MPH, Bruce M. Psaty, MD, PhD, Eric B. Rimm, ScD, Rozenn N. \\ Lemaitre, PhD, MPH, Gregory L. Burke, MD, MS, Mary F. Lyles, MD, David Lefkowitz, MD, and \\ David S. Siscovick, MD, MPH \\ From the Channing Laboratory, Brigham and Women's Hospital and Harvard Medical School, and \\ Departments of Epidemiology and Nutrition, Harvard School of Public Health, Boston, Mass (D.M., E.B.R.); \\ Cardiovascular Health Research Unit, Departments of Medicine (B.M.P, R.N.L., D.S.S.) and Epidemiology \\ (B.M.P., D.S.S.), University of Washington, Seattle, Wash; and Departments of Public Health Sciences \\ (G.L.B.), Medicine (M.F.L.), and Neurology (D.L.), Wake Forest University School of Medicine, Winston- \\ Salem, NC.
}

\section{Abstract}

Background-Atrial fibrillation (AF) is the most common arrhythmia in clinical practice and is particularly common in the elderly. Although effects of fish intake, including potential antiarrhythmic effects, may favorably influence risk of AF, relationships between fish intake and $\mathrm{AF}$ incidence have not been evaluated.

\begin{abstract}
Methods and Results_- In a prospective, population-based cohort of 4815 adults $\geq$ age 65 years, usual dietary intake was assessed at baseline in 1989 and 1990. Consumption of tuna and other broiled or baked fish correlated with plasma phospholipid long-chain n-3 fatty acids, whereas consumption of fried fish or fish sandwiches (fish burgers) did not. AF incidence was prospectively ascertained on the basis of hospital discharge records and annual electrocardiograms. During 12 years' followup, 980 cases of incident AF were diagnosed. In multivariate analyses, consumption of tuna or other broiled or baked fish was inversely associated with incidence of AF, with $28 \%$ lower risk with intake 1 to 4 times per week (HR=0.72, 95\% $\mathrm{CI}=0.58$ to $0.91, P=0.005)$, and $31 \%$ lower risk with intake $\geq 5$ times per week ( $\mathrm{HR}=0.69,95 \% \mathrm{CI}=0.52$ to $0.91, P=0.008)$, compared with $<1$ time per month $(P$ trend $=0.004)$. Results were not materially different after adjustment for preceding myocardial infarction or congestive heart failure. In similar analyses, fried fish/fish sandwich consumption was not associated with lower risk of AF.
\end{abstract}

Conclusions__ Among elderly adults, consumption of tuna or other broiled or baked fish, but not fried fish or fish sandwiches, is associated with lower incidence of AF. Fish intake may influence risk of this common cardiac arrhythmia.

\section{Keywords}

fibrillation, atrial; diet; fish; aging; nutrition

A trial fibrillation (AF) is the most common cardiac arrhythmia, affecting more than 2 million individuals in the United States. ${ }^{1}$ Risk increases markedly with advancing age. ${ }^{1-3}$ After age 65 years, the incidence of $\mathrm{AF}$ is approximately $2 \%$ per year, with risk factors including older age; presence of valvular or coronary heart disease; greater systolic blood pressure, left atrial size, serum glucose, and C-reactive protein levels; and lower alcohol use, serum cholesterol, and forced expiratory volume in 1 second. ${ }^{3-4}$ AF results in significant morbidity, including fatigue, reduced exercise tolerance, increased stroke risk, and potential bleeding complications from anticoagulant therapy. 1,5 
Little is known about relationships of nutritional factors with incidence of AF. Fish intake may lower blood pressure, reduce systemic inflammation, and improve left ventricular diastolic function, ${ }^{6-16}$ each of which could favorably affect risk of AF. Fish consumption also appears to reduce risk of fatal ventricular arrhythmias, consistent with antiarrhythmic effects of longchain $n-3$ polyunsaturated fatty acids in fish. ${ }^{17-24}$ In cultured rat atrial myocytes, $n-3$ fatty acids reduce induced asynchronous contractile activity, 25 suggesting that $n-3$ fatty acids from fish may have antiarrhythmic effects on atrial muscle. However, it is unknown if fish intake - or indeed any dietary factor-influences risk of AF. We therefore investigated relationships between fish consumption and risk of AF in the Cardiovascular Health Study (CHS), a population-based, prospective cohort study of determinants of cardiovascular risk among older adults. Our primary hypothesis was that intake of tuna or other broiled or baked fish would be associated with lower incidence of AF.

\section{Methods}

\section{Design and Population}

The design and recruitment process of CHS have been described. ${ }^{26-27}$ Briefly, 5201 men and women $\geq$ age 65 years were randomly selected and enrolled from Medicare eligibility lists in 4 US communities in 1989 and 1990; an additional 687 black participants enrolled in 1992 were not included in this analysis because a food frequency questionnaire (FFQ) was not administered to these subjects at baseline. Each center's institutional review committee approved the study, and all subjects gave informed consent. Mean age at enrollment was 72.8 years (range, 65 to 100). We excluded 284 participants with prevalent $\mathrm{AF}$ at baseline ${ }^{28}$ and 102 participants with incomplete data on fish consumption, resulting in 4815 participants included in this analysis. At baseline, participants responded to questionnaires on health status, medical history, and cardiovascular risk factors and underwent standardized clinical examination, resting 12-lead ECG, 2-dimensional echocardiography, pulmonary function testing, and laboratory evaluation. $2,3,26-29$

\section{Dietary Assessment}

Usual dietary intake was assessed at baseline with the use of a picture-sort version of the National Cancer Institute FFQ. ${ }^{30}$ Participants were asked to indicate how often, on average, they had consumed various specific foods during the past year, including tuna fish, other broiled or baked fish, and fried fish or fish sandwiches (fish burgers). Macronutrient intakes were estimated from questionnaire responses and adjusted for total caloric intake by using regression analyses. ${ }^{31-32}$ When macronutrients were not estimated because of missing FFQ responses $(n=500)$, analyses were performed with a missing indicator category. As a biomarker of $n-3$ fatty acid content, we have previously evaluated correlations of fish intake with plasma phospholipid eicosapentaenoic acid (EPA; 20:5n-3) and docosahexaenoic acid (DHA; 22:6n-3) in a subset of participants. ${ }^{22}$ Combined EPA+DHA levels correlated with intake of tuna fish (Spearman correlation $=0.35, P<0.01)$ and other fish $(r=0.59, P<0.001)$ but not fried fish or fish sandwiches $(r=0.04, P=0.78)$, consistent with lean types of fish typically fried (cod, pollock, etc).

\section{Identification of AF}

Participants were followed up via annual examinations, including annual resting 12-lead ECGs through year 9 and interim 6-month telephone contacts. ${ }^{33}$ Hospital records were obtained for all hospitalizations, with adjudication of cardiovascular events by centralized events committees. ${ }^{33}$ Cases of AF were identified by (1) annual 12-lead ECGs, centrally reviewed at the CHS ECG Reading Center ${ }^{34}$ or (2) hospital discharge diagnoses (International Classification of Diseases, 9th Revision [ICD 9] codes 427.3, 427.31, or 427.32). Review of medical records (including hospital ECGs) in a subset of cases demonstrated that hospital 
discharge diagnoses have an accuracy (positive predictive value) of $98.6 \%$ for diagnosing $\mathrm{AF}$ in CHS. ${ }^{3}$ To further evaluate the potential for missed outcomes, we examined the results of 24-hour Holter monitoring performed at year 5 in a subset of 819 participants. Fifteen individuals demonstrated sustained $\mathrm{AF}$, all of whom were identified by the above criteria, and 4 individuals demonstrated intermittent AF, 3 of whom were identified by the above criteria. Thus, as determined by 24-hour Holter monitoring, only 1 in 819 individuals $(0.1 \%)$ had sustained or intermittent AF not identified by the above criteria.

\section{Statistical Analysis}

Cox proportional-hazards models were used to estimate risk, censoring at first event (earliest date of ECG or hospital discharge diagnosis of AF), death, or last day of follow-up through June 30, 2001. Intakes of tuna and other broiled or baked fish were correlated with each other $(r=0.36, P<0.001)$ and with $\mathrm{n}-3$ fatty acids as described above. Tuna consumption and other fish consumption were therefore evaluated together, summing median intakes of their respective response categories (combined correlation with $\mathrm{EPA}+\mathrm{DHA}=0.55, P<0.001$ ). Fried fish/fish sandwich consumption was modestly positively correlated with tuna/other fish intake $(r=0.14)$ and was evaluated separately. To minimize potential confounding, covariates were selected on the basis of clinical interest, published associations with $\mathrm{AF}, 2,3,25$ or associations with exposures or outcomes in the current cohort. Three multivariate models are presented: (1) adjusted for age, gender, race (white, non-white), education (<high school, high school, >high school), diabetes mellitus, body mass index, prevalent coronary heart disease, prevalent valvular disease, smoking status (never, former, current), pack-years of smoking, leisure-time activity, tuna/other fish intake or fried fish/fish sandwich intake, and total caloric intake; (2) further adjusted for other dietary factors (alcohol, saturated fat, beef/pork, fruits, vegetables, cereal fiber); and (3) further adjusted for potential confounders or mediators (systolic blood pressure, diastolic blood pressure, left ventricular systolic function [normal, borderline/ abnormal], treated hypertension, C-reactive protein). We also evaluated potential mediation by intermediary myocardial infarction (MI) or congestive heart failure (CHF) by using timevarying adjustment and stratified analyses. Other covariates did not materially alter the relations between fish intake and AF risk and were therefore excluded from the final models, including study site, income, exercise intensity, prevalent stroke or transient ischemic attack, and prevalent asthma or emphysema; use of $\beta$-blockers, lipid-lowering medication, fish oil, and estrogen; echo-determined left atrial size and left ventricular mass; forced expiratory volume in 1 second; fasting glucose, insulin, LDL, HDL, triglycerides, and fibrinogen; and estimated intake of total fat, carbohydrates, protein, linolenic acid, fiber, wine, thiamine, vitamin A, and vitamin C. Kaplan-Meier survival methods were used to evaluate AF-free survival according to tuna/other fish and fried fish/fish sandwich consumption (each adjusted for the other). Intake categories were entered as ordinal variables in tests for trend and for evaluation of differences in baseline characteristics using linear (continuous variables) or logistic (dichotomous variables) regression. Likelihood-ratio testing compared models with and without multiplicative interaction terms to assess effect modification by age, gender, education, prevalent coronary heart disease, treated hypertension, systolic blood pressure, and study site. All probability values are 2 tailed $(\alpha=0.05)$. Analyses were performed with the use of Stata 8.0 (College Station, Tex).

\section{Results}

Mean fried fish/fish sandwich and tuna/other fish consumption were 0.7 and 2.2 servings per week, respectively. Tuna/other fish consumption was associated with younger age, female gender, and higher education, whereas fried fish/fish sandwich consumption was associated with male gender, nonwhite race, and lower education (Table 1). Tuna/other fish consumption was generally associated with a more favorable cardiovascular risk profile, although tuna/other 
fish intake was also associated with higher LDL cholesterol. Tuna/other fish consumption was inversely associated with saturated fat intake and positively associated with intake of alcohol, fruits, vegetables, and cereal fiber; fried fish/fish sandwich consumption was positively associated with intake of saturated fat, beef/pork, and vegetables. As noted previously, in a subsample of participants, greater tuna/other fish intake but not fried fish/fish sandwich intake was associated with higher plasma phospholipid EPA+DHA.

During 12 years' follow-up, 980 participants developed incident AF. Of these, 739 persons (75\%) were diagnosed by hospital records, $54(6 \%)$ by annual ECG, and 187 (19\%) by both methods. Figure 1 presents AF-free survival according to fish consumption. Among persons consuming tuna/other fish $\geq 5$ times per week, AF incidence was 19 per 1000 person-years, compared with 33 per 1000 person-years with intake $<1$ time per month $(P<0.0001)$. Among persons consuming fried fish/fish sandwich $\geq 1$ time per week, AF incidence was 27 per 1000 person-years, compared with 22 per 1000 person-years with intake $<1$ time per month $(P=0.0009)$.

Age- and multivariate-adjusted risks are presented in Table 2. After adjustment for potential confounders (Model 1), there was 24\% lower risk with tuna/other fish intake 1 to 3 times per month $(\mathrm{HR}=0.76,95 \% \mathrm{CI}=0.61$ to $0.95, P=0.02), 30 \%$ lower risk with intake 1 to 4 times per week $(\mathrm{HR}=0.70,95 \% \mathrm{CI}=0.57$ to $0.87, P=0.001)$, and $35 \%$ lower risk with intake $\geq 5$ times per week $(\mathrm{HR}=0.65,95 \% \mathrm{CI}=0.51$ to $0.84, P=0.001)$, compared with $<1$ time per month $(P$ trend=0.001). Results were not greatly altered by further adjustment for other dietary factors (Model 2) or other potential confounders/mediators (Model 3).

In contrast, fried fish/fish sandwich consumption was not associated with lower AF risk (Table 2). After adjustment for potential confounders (Model 1), intake $\geq 1$ time per week was associated with $24 \%$ higher risk $(\mathrm{HR}=1.24,95 \% \mathrm{CI}=1.03$ to $1.49, P=0.03)$ compared with $<1$ time month. This positive association was attenuated by adjustment for other dietary factors (Model 2) and potential confounders/mediators (Model 3). Of potential mediators, adjustment for baseline left ventricular systolic function, treated hypertension, and C-reactive protein levels appeared to have the greatest effect (not shown).

Exclusion of participants taking fish oil supplements $(n=183)$ or with prevalent coronary heart disease, stroke, or transient ischemic attack at baseline $(n=1018)$ did not materially alter results (not shown). There was little evidence for interaction between tuna/other fish and fried fish/ fish sandwich consumption ( $P$ for interaction $>0.05$ ) (Figure 2 ). There was also little evidence that findings varied according to age, gender, education, prevalent coronary heart disease, treated hypertension, systolic blood pressure, or study site ( $P>0.05$ for each interaction).

Because AF may occur as a result of preceding MI or CHF, we also evaluated the extent to which observed relationships between fish intake and AF might be mediated by MI or CHF (Table 3). After adjustment for MI or CHF as time-varying covariates, relationships between tuna/other fish intake and AF incidence were not greatly altered, with $29 \%$ lower risk with consumption 1 to 4 times per week $(\mathrm{HR}=0.71,95 \% \mathrm{CI}=0.55$ to $0.88, P=0.001)$ and $31 \%$ lower risk with consumption $\geq 5$ times per week $(\mathrm{HR}=0.69,95 \% \mathrm{CI}=0.54$ to $0.89, P=0.004)$ compared with $<1$ time per month $(P$ trend=0.003). Findings were similar among persons without MI or $\mathrm{CHF}$ or evaluating only persons with prevalent $\mathrm{MI}$ or $\mathrm{CHF}$, although confidence intervals for intake $\geq 5$ times per week included unity due to fewer numbers of events. In similar analyses, relationships between fried fish/fish sandwich intake and AF risk were not greatly altered by adjustment for preceding MI or CHF; however, associations appeared attenuated among persons without MI or CHF (Table 3). 


\section{Discussion}

In this population-based, prospective study among elderly adults, greater consumption of tuna or other broiled or baked fish was associated with lower risk of incident AF. This observed relationship does not prove causality and may be related to other factors associated with both fish intake and risk of AF. However, the association persisted after adjustment for a variety of demographic, clinical, lifestyle, laboratory, and dietary characteristics, including preceding MI and CHF. These results suggest that regular intake of tuna or other broiled or baked fish may be a simple and important deterrent of AF among older men and women.

It is biologically plausible that this observed relationship is due to effects of long-chain n-3 fatty acids in fatty fish. In experimental studies, fish oil has favorable effects on several hemodynamic, vascular, and left ventricular indices. In rats, intake of fish oil reduces peripheral vascular resistance, ${ }^{6}$ reduces myocardial oxygen consumption at any given workload, ${ }^{7}$ reduces markers of ischemia and increases contractile recovery after ischemia and reperfusion, ${ }^{7}$ and reduces left ventricular hypertrophy in hypertensive animals. ${ }^{8}$ In nonhuman primates, fish oil enhances left ventricular diastolic function. ${ }^{9-10}$ In humans, fish oil supplementation lowers blood pressure, ${ }^{11}$ reduces markers of systemic inflammation, ${ }^{12}$ attenuates vasoconstrictive responses to angiotensin II, ${ }^{13-14}$ improves arteriolar wall compliance, ${ }^{15}$ and improves left ventricular diastolic filling. ${ }^{16}$ Considerable epidemiological, clinical trial, and animalexperimental evidence also points to antiarrhythmic effects of n-3 fatty acids in fish, ${ }^{17-24}$ which decrease excitability and cytosolic calcium fluctuations of ventricular myocytes via inhibition of $\mathrm{Na}^{+}$and L-type $\mathrm{Ca}^{++}$channels. ${ }^{24}$ In vitro, $\mathrm{n}-3$ fatty acids also reduce induced asynchronous contractile activity of atrial myocytes, ${ }^{25}$ suggesting that antiarrhythmic effects may extend to atrial myocardium. Each of these effects may plausibly reduce risk of AF.

We did not observe lower risk of AF with intake of fried fish or fish sandwiches, which generally are made from lean fish and did not correlate with n-3 fatty acid levels; rather, we observed a positive association. This observed higher risk might be a result of residual confounding, for example, from other lifestyle or dietary factors. The preparation method might also influence risk. Frying can greatly alter a fish meal's nutrient composition, increasing contents of n-6 fatty acids, trans-fatty acids, and oxidation products, particularly when oils are used repeatedly for frying. ${ }^{35-36}$ Adjustment for baseline coronary heart disease, left ventricular systolic function, blood pressure, treated hypertension, and C-reactive protein levels attenuated the higher AF risk seen with fried fish/fish sandwich intake, which suggests that these factors may in part confound or mediate the association. Further investigation is necessary to confirm these observations, determine if they are specific for certain kinds of fish or frying oils, and examine potential mechanisms and alternative explanations. Our findings suggest, however, that intake of fried fish or fish sandwiches is unlikely to reduce risk of AF.

Our analysis has several strengths. The prospective design reduces potential bias from recall differences. The population-based recruitment strategy enhances generalizability.

Standardized assessment of a wide variety of participant characteristics increases the capacity to adjust for confounding. Close follow-up, annual ECGs, and review of all hospitalizations reduce potential for missed or misclassified outcomes.

There are also limitations to our findings. Fish intake was assessed at baseline, and consumption may have changed over time; this likely would cause underestimation of associations between fish intake and AF. Cases of paroxysmal AF may have been missed, particularly if asymptomatic. The possibility of residual confounding due to unmeasured or imprecisely measured factors cannot be excluded. The associations were observed in older individuals participating in a cohort study and may not be generalizable to other populations. 
Our findings indicate that consumption of tuna and other broiled or baked fish, but not fried fish or fish sandwiches, is associated with lower incidence of AF among older adults. These results suggest that fish intake may influence the risk of this common cardiac arrhythmia. Confirmation of these findings in other studies and evaluation of potential mechanisms of effect, such as effects on blood pressure, left ventricular diastolic function, systemic inflammation, or direct antiarrhythmic effects, are indicated.

\section{Acknowledgements}

Research reported in this article was supported by contracts N01-HC-85079 through N01-HC-85086, N01-HC-35129, and N01-HC-15103 from the National Heart, Lung, and Blood Institute. Dr Mozaffarian was supported in part by a National Research Service Award Training Grant in Academic Nutrition (DK07703). For a full list of CHS investigators/institutions, see "About CHS-Principal Investigators and Study Sites" at http://chs-nhlbi.org.

\section{References}

1. American Heart Association. Heart Disease and Stroke Statistics-2004 Update Dallas, Tex: American Heart Association; 2003.

2. Furberg CD, Psaty BM, Manolio TA, et al. Prevalence of AF in elderly subjects-CHS. Am J Cardiol 1994;74:236-241. [PubMed: 8037127]

3. Psaty BM, Manolio TA, Kuller LH, et al. Incidence of and risk factors for AF in older adults. Circulation 1997;96:2455-2461. [PubMed: 9337224]

4. Aviles RJ, Martin DO, Apperson-Hansen C, et al. Inflammation as a risk factor for AF. Circulation 2003;108:3006-3010. [PubMed: 14623805]

5. Movsowitz HD, Lampert C, Jacobs LE, et al. Atrial fibrillation in the elderly. Am J Geriatr Cardiol 1994;3:26-40. [PubMed: 11416307]

6. Demaison L, Blet J, Sergiel JP, et al. Effect of dietary polyunsaturated fatty acids on contractile function of hearts isolated from sedentary and trained rats. Reprod Nutr Dev 2000;40:113-125. [PubMed: 10863956]

7. Pepe S, McLennan PL. Cardiac membrane fatty acid composition modulates myocardial oxygen consumption and postischemic recovery of contractile function. Circulation 2002;105:2303-2308. [PubMed: 12010914]

8. von Au D, Brandle M, Rupp H, et al. Influence of diet rich in fish oil on blood pressure, body weight and cardiac hypertrophy in spontaneously hypertensive rats. Eur J Appl Physiol Occup Physiol 1988;58:97-99. [PubMed: 2974415]

9. Charnock JS, McLennan PL, Abeywardena MY. Dietary modulation of lipid metabolism and mechanical performance of the heart. Mol Cell Biochem 1992;116:19-25. [PubMed: 1480148]

10. McLennan PL, Barnden LR, Bridle TM, et al. Dietary fat modulation of left ventricular EF in the marmoset due to enhanced filling. Cardiovasc Res 1992;26:871-877. [PubMed: 1451164]

11. Geleijnse JM, Giltay EJ, Grobbee DE, et al. Blood pressure response to fish oil supplementation: metaregression analysis of randomized trials. J Hypertens 2002;20:1493-1499. [PubMed: 12172309]

12. James MJ, Gibson RA, Cleland LG. Dietary polyunsaturated fatty acids and inflammatory mediator production. Am J Clin Nutr 2000;71:343S-348S. [PubMed: 10617994]

13. Chin JP, Gust AP, Nestel PJ, et al. Marine oils dose-dependently inhibit vasoconstriction of forearm resistance vessels in humans. Hypertension 1993;21:22-28. [PubMed: 8418020]

14. Kenny D, Warltier DC, Pleuss JA, et al. Effect of omega-3 fatty acids on the vascular response to angiotensin in normotensive men. Am J Cardiol 1992;70:1347-1352. [PubMed: 1442589]

15. McVeigh GE, Brennan GM, Cohn JN, et al. Fish oil improves arterial compliance in NIDDM. Arterioscler Thromb 1994;14:1425-1429. [PubMed: 8068603]

16. Grimsgaard S, Bonaa KH, Hansen JB, et al. Effects of highly purified EPA and DHA on hemodynamics in humans. Am J Clin Nutr 1998;68:52-59. [PubMed: 9665096]

17. Siscovick DS, Raghunathan TE, King I, et al. Dietary intake and cell membrane levels of long-chain n-3 polyunsaturated fatty acids and risk of primary cardiac arrest. JAMA 1995;274:1363-1367.

[PubMed: 7563561] 
18. Albert CM, Hennekens CH, O'Donnell CJ, et al. Fish consumption and risk of sudden cardiac death. JAMA 1998;279:23-28. [PubMed: 9424039]

19. GISSI-Investigators. Dietary supplementation with n-3 polyunsaturated fatty acids and vitamin $E$ after myocardial infarction: the GISSI-Prevenzione trial. Lancet 1999;354:447-455. [PubMed: 10465168]

20. Albert CM, Campos H, Stampfer MJ, et al. Blood levels of long-chain n-3 fatty acids and risk of sudden death. N Engl J Med 2002;346:1113-1118. [PubMed: 11948270]

21. Lemaitre RN, King I, Mozaffarian D, et al. Plasma phospholipid n-3 polyunsaturated fatty acids, fatal ischemic heart disease, and nonfatal MI in older adults-CHS. Am J Clin Nutr 2003;77:319-325. [PubMed: 12540389]

22. Mozaffarian D, Lemaitre RN, Kuller LH, et al. Cardiac benefits of fish consumption may depend on the type of fish meal consumed-CHS. Circulation 2003;107:1372-1377. [PubMed: 12642356]

23. McLennan PL. Myocardial membrane fatty acids and antiarrhythmic actions of dietary fish oil in animal models. Lipids 2001;36:111S-114S.

24. Kang JX, Leaf A. Prevention of fatal cardiac arrhythmias by polyunsaturated fatty acids. Am J Clin Nutr 2000;71:202S-207S. [PubMed: 10617972]

25. Jahangiri A, Leifert WR, Patten GS, et al. Termination of asynchronous contractile activity in rat atrial myocytes by n-3 polyunsaturated fatty acids. Mol Cell Biochem 2000;206:33-41. [PubMed: 10839192]

26. Fried LP, Borhani NO, Enright P, et al. Cardiovascular Health Study: design and rationale. Ann Epidemiol 1991;1:263-276. [PubMed: 1669507]

27. Tell GS, Fried LP, Hermanson B, et al. Recruitment of adults 65 years and older as participants in CHS. Ann Epidemiol 1993;3:358-366. [PubMed: 8275211]

28. Psaty BM, Kuller LH, Bild D, et al. Methods of assessing prevalent cardiovascular disease in CHS. Ann Epidemiol 1995;5:270-277. [PubMed: 8520708]

29. Cushman M, Cornell ES, Howard PR, et al. Laboratory methods and quality assurance in CHS. Clin Chem 1995;41:264-270. [PubMed: 7874780]

30. Kumanyika SK, Tell GS, Shemanski L, et al. Dietary assessment using a picture-sort approach. Am J Clin Nutr 1997;65:1123S-1129S. [PubMed: 9094908]

31. Smucker R, Block G, Coyle L, et al. Dietary and risk factor questionnaire and analysis system for personal computers. Am J Epidemiol 1989;129:445-449. [PubMed: 2912053]

32. Willett W, Stampfer MJ. Total energy intake-implications for epidemiologic analyses. Am J Epidemiol 1986;124:17-27. [PubMed: 3521261]

33. Ives DG, Fitzpatrick AL, Bild DE, et al. Surveillance and ascertainment of cardiovascular eventsCHS. Ann Epidemiol 1995;5:278-285. [PubMed: 8520709]

34. Rautaharju PM, MacInnis PJ, Warren JW, et al. Methodology of ECG interpretation in the Dalhousie program-NOVACODE ECG classification procedures. Methods Inf Med 1990;29:362-374. [PubMed: 2233384]

35. Candela M, Astiasaran I, Bello J. Deep-fat frying modifies high-fat fish lipid fraction. J Agric Food Chem 1998;46:2793-2796.

36. Williams MJ, Sutherland WH, McCormick MP, et al. Impaired endothelial function following meal rich in used cooking fat. J Am Coll Cardiol 1999;33:1050-1055. [PubMed: 10091835] 

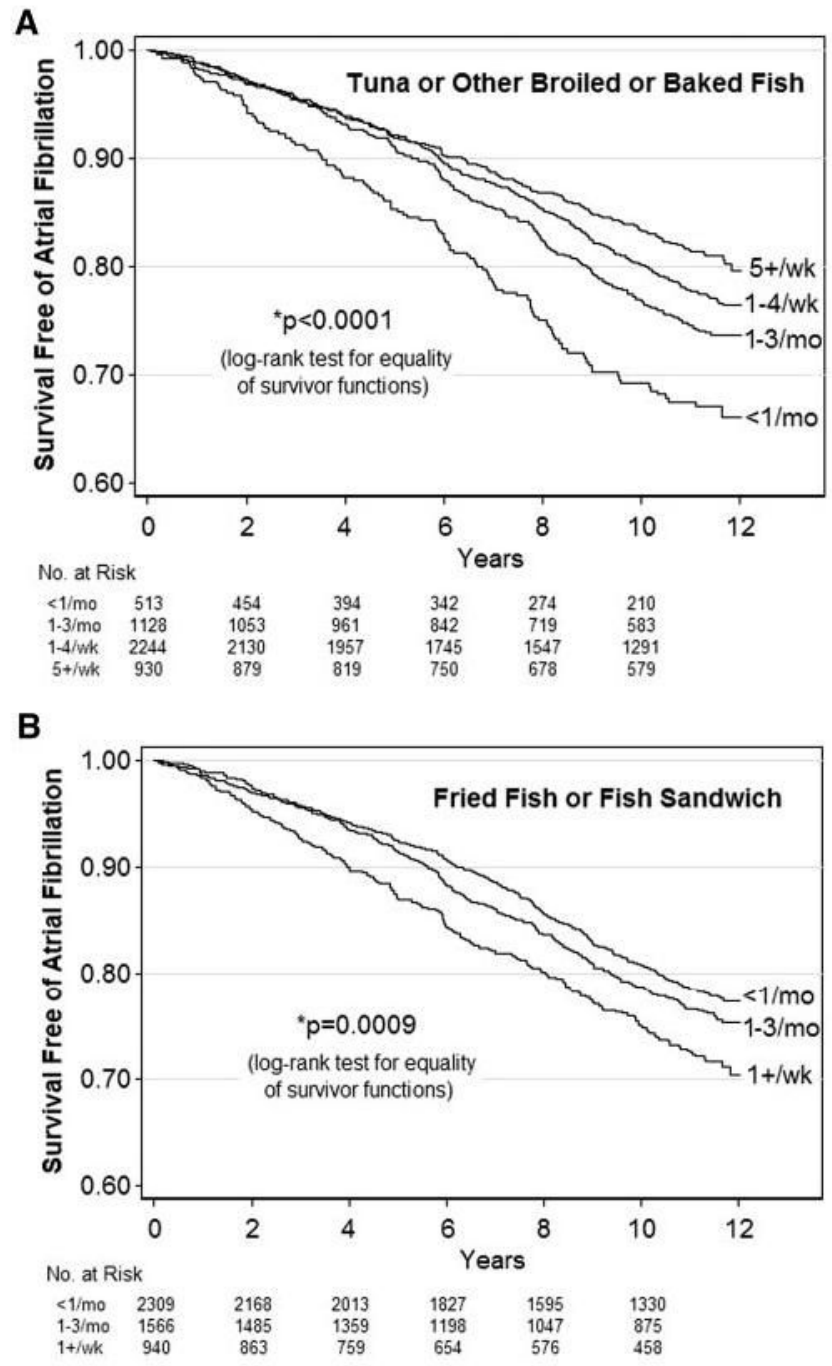

Figure 1.

AF-free survival according to fish consumption. 


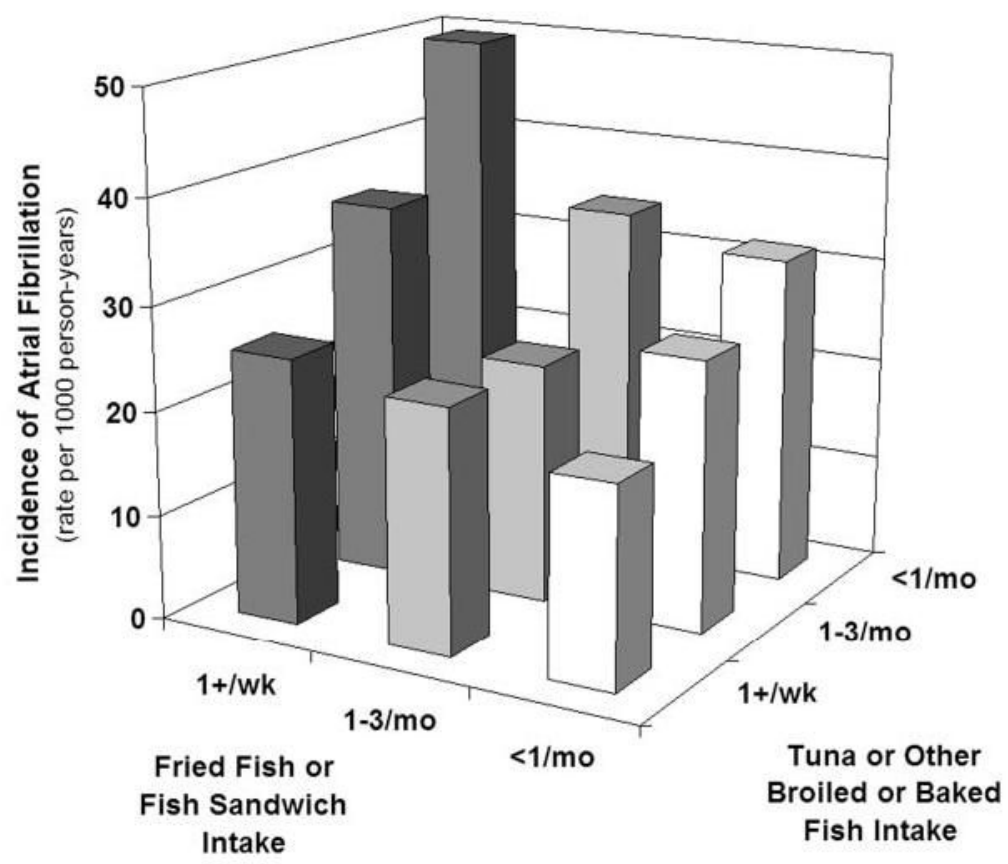

Figure 2.

AF incidence according to both tuna/other fish and fried fish/fish sandwich consumption. For groups consuming tuna/other fish $\geq 1$ /week, AF cases/numbers at risk were 161/781, 213/1031, and 234/1362 for fried fish/fish sandwich intake $\geq 1 /$ week, $1-3 /$ month, and $<1 /$ month, respectively; for groups consuming tuna/other fish $1-3 /$ month, the corresponding numbers were 29/99, 78/416, and 134/613; and for groups consuming tuna/other fish $<1 /$ month, the corresponding numbers were 20/60, 32/119, and 79/334. 


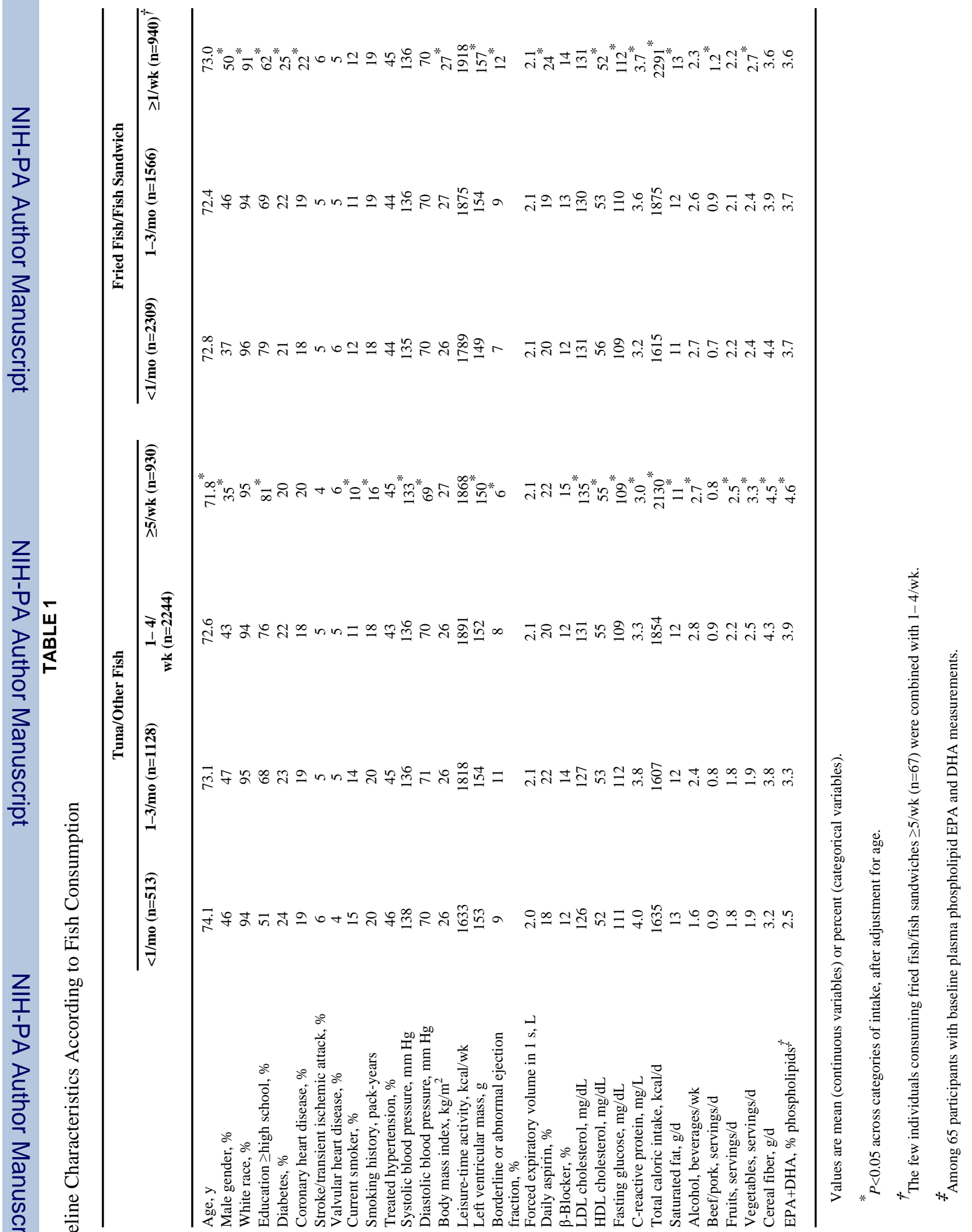


TABLE 2

Risk of Incident AF According to Fish Consumption

\begin{tabular}{|c|c|c|c|c|c|}
\hline & \multicolumn{4}{|c|}{ Frequency of Fish Consumption } & \multirow[b]{2}{*}{$P$ trend } \\
\hline & $<1 /$ mo & $1-3 / \mathrm{mo}$ & $1-4 / w k$ & $\geq 5 / w k$ & \\
\hline Tuna/other fish, $\mathrm{n}$ & 513 & 1128 & 2244 & 930 & \\
\hline No. events & 131 & 241 & 444 & 164 & \\
\hline Person-years & 3952 & 9728 & 20220 & 8644 & \\
\hline \multicolumn{6}{|l|}{ HR (95\% CI): } \\
\hline Age adjusted & 1.0 (referent) & $0.78(0.63-0.97)$ & $0.71(0.58-0.87)$ & $0.65(0.52-0.82)$ & $<0.001$ \\
\hline Model $1 *$ & 1.0 (referent) & $0.76(0.61-0.95)$ & $0.70(0.57-0.87)$ & $0.65(0.51-0.84)$ & 0.001 \\
\hline Model $2^{\dagger}$ & 1.0 (referent) & $0.82(0.66-1.04)$ & $0.72(0.58-0.91)$ & $0.69(0.52-0.91)$ & 0.004 \\
\hline Model $3^{*}$ & 1.0 (referent) & $0.81(0.65-1.03)$ & $0.72(0.57-0.90)$ & $0.70(0.53-0.93)$ & 0.007 \\
\hline Fried fish/fish & 2309 & 1566 & 940 & $\S$ & \\
\hline $\begin{array}{l}\text { sandwich, } \mathrm{n} \\
\text { No. events }\end{array}$ & 447 & 323 & 210 & & \\
\hline Person-years & 20787 & 13955 & 7803 & & \\
\hline \multicolumn{6}{|l|}{ HR $(95 \%$ CI): } \\
\hline Age adjusted & 1.0 (referent) & $1.12(0.97-1.29)$ & $1.27(1.08-1.49)$ & & 0.004 \\
\hline Model $1 *$ & 1.0 (referent) & $1.07(0.92-1.25)$ & $1.24(1.03-1.49)$ & & 0.03 \\
\hline Model $2^{\dagger}$ & 1.0 (referent) & $1.01(0.86-1.19)$ & $1.22(1.00-1.49)$ & & 0.08 \\
\hline Model $3^{\ddagger}$ & 1.0 (referent) & $0.96(0.82-1.13)$ & $1.17(0.96-1.43)$ & & 0.23 \\
\hline
\end{tabular}

* Includes age, gender, race, education, diabetes mellitus, body mass index, prevalent coronary heart disease, prevalent valvular heart disease, smoking status, pack-years of smoking, leisure-time activity, tuna/other fish intake, fried fish/fish sandwich intake, and total caloric intake.

${ }^{\dagger}$ Further adjusted for other dietary factors (alcohol, saturated fat, beef/pork, fruits, vegetables, cereal fiber).

Further adjusted for potential confounders/mediators (systolic blood pressure, diastolic blood pressure, left ventricular systolic function at baseline, treated hypertension, C-reactive protein).

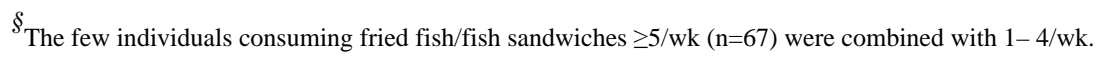




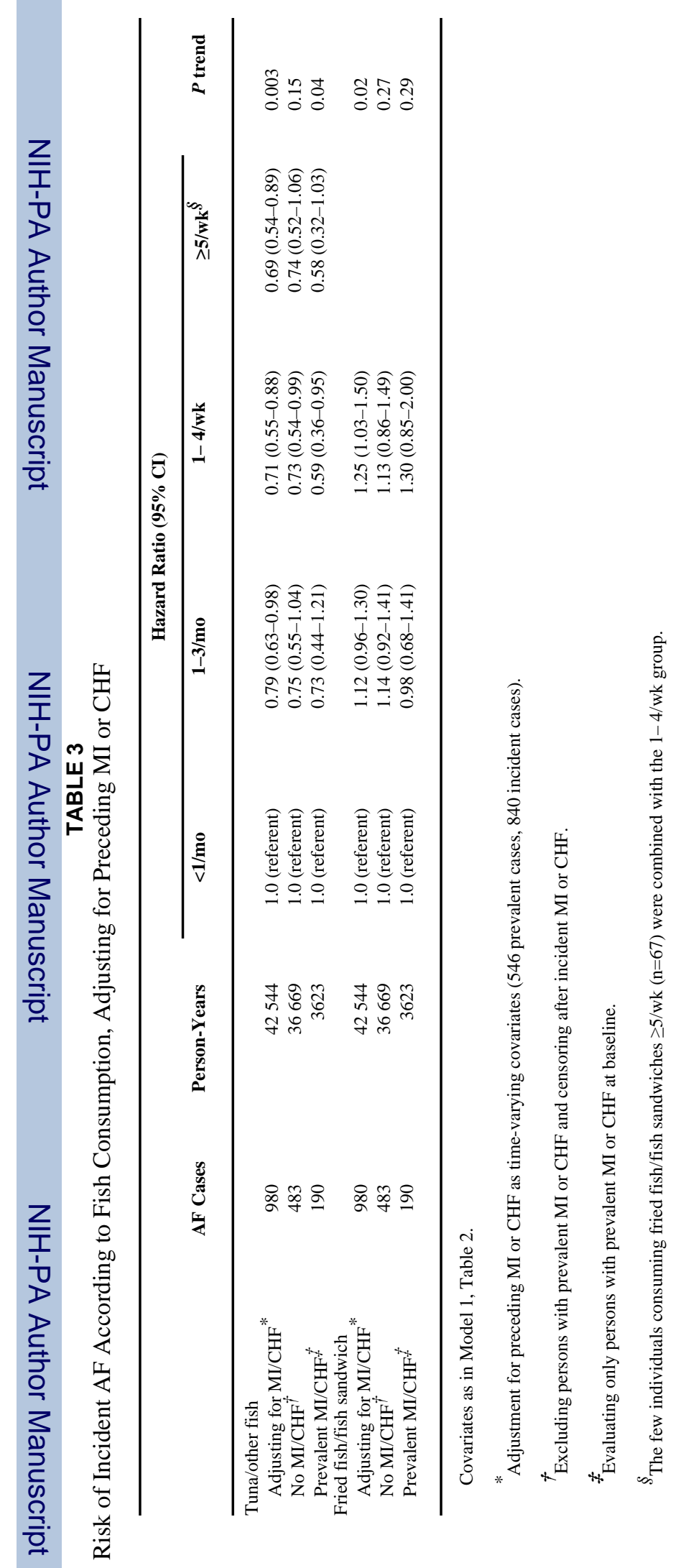

